\title{
Psychosomatic Symptoms, Smoking and Binge Drinking in Adolescence: a Nationwide Study in Greece

\section{Abstract}

Aim: The present study aimed to investigate differences in smoking and binge drinking between adolescents with high and low levels of psychosomatic symptoms and explore the associations of adolescent smoking and binge drinking with self-reported psychosomatic symptoms in a large nationwide random school-based sample of Greek adolescents aged 12-18 years.

Method: Adolescents with high levels of psychosomatic symptoms were compared in terms of smoking, binge drinking, gender, age and socio-economic status to their peers with lower levels of psychosomatic symptoms. In order to evaluate the multiple effects of adolescents' smoking, binge drinking and socio-demographic features on psychosomatic symptoms, a multiple logistic regression model was constructed.

Results: Adolescents with smoking and/or binge drinking practices reported higher levels of psychosomatic symptoms compared to peers with no experiences of such health-risk behaviours. Moreover, adolescents with smoking and/or binge drinking practices had an amplified risk for high levels of psychosomatic symptoms, revealing a significantly impaired psychosomatic functioning compared to abstainers.

Conclusions: Professionals in school and clinical settings should be cautious for smoking and binge drinking practices when assessing adolescents with high levels of psychosomatic symptoms, so as early identification of at-risk individuals and timely, appropriate care planning is facilitated.
George Giannakopoulos ${ }^{1}$, Dimitra Petanidou²,

Chara Tzavara², Christine Dimitrakaki², Gerasimos Kolaitis ${ }^{1}$, Yannis Tountas ${ }^{2}$

1 Department of Child Psychiatry, Athens University Medical School, Aghia Sophia Children's Hospital, Athens, Greece.

2 Centre for Health Services Research, Department of Hygiene, Epidemiology and Medical Statistics, Athens University Medical School, Athens, Greece.

\section{Contact information:}

\section{George Giannakopoulos.}

Department of Child Psychiatry, Athens University Medical School, Aghia Sophia Children's Hospital, Thivon \& Papadiamantopoulou 11527, Athens, Greece.

झ giannakopoulos.med@gmail.com 


\section{Introduction}

Adolescence has been considered a key formative period for health, since it is during this phase of human development that individuals enhance their self-awareness regarding their health and adopt health behaviours and lifestyle patterns that may extend into later life. Research evidence has suggested that psychosomatic symptoms (e.g. headaches, backaches, irritability, difficulties in getting to sleep) and health-compromising behaviours are the first indicators of problems in both psychological and social development during adolescence [1]. Research exploring the relationship of psychosomatic symptoms with common health-risk behaviours, such as tobacco and alcohol use, is still meager [2, 3], even though international studies have documented alarming findings regarding the prevalence of psychosomatic symptoms, tobacco and excessive alcohol use among adolescents [4]. The aim of the present study was to explore the associations of adolescents' psychosomatic symptoms with smoking and binge drinking behaviors in a large, nation-wide, school-based sample.

\section{Methods}

This study was conducted within the framework of the European project "Screening and Promotion for Health Related Quality of Life (HRQoL) in Children and Adolescents: A European Public Health Perspective", (acronym: KIDSCREEN). A thorough description of the KIDSCREEN study has been provided elsewhere [5]. A sample of 1,900 adolescents aged 12 to 18-years-old was recruited, while a total of 1194 (63\% response rate) self-reported questionnaires were returned. Data from 1163 adolescents without missing values were analysed in the present study. Ethical approval was attained from the National Ministry of Education. The KIDSCREEN questionnaires were accompanied by the parents' information letter, an informed consent form, and the information letter for the students.
The consent to participate was obtained before survey administration.

Psychosomatic symptoms were measured through the Health Behaviour in School-aged Children Symptom Checklist (HBSC-SCL) [6], a selfadministered brief screening instrument indicating the frequency of occurrence of eight common somatic and psychological complaints (i.e. headache, stomachache, backache, dizziness, feeling low, irritability or bad temper, feeling nervous, and difficulties in getting to sleep). Ratings ranged from "about every day" (5) to "rarely/never" (1). Adolescents who reported at least two symptoms more than once a week -corresponding to scoring categories 4 and 5- were categorized as having high levels of psychosomatic symptoms, according to previous relevant research $[3,4,7,8]$. Adolescents who reported less frequent and fewer and/or no experiences of psychosomatic symptoms-corresponding to scoring categories 1, 2 and 3- were grouped as having low levels of psychosomatic symptoms (Cronbach's alpha $=0.79$ ). The HBSC$\mathrm{SCL}$ has shown adequate psychometric properties and has been commonly used to assess psychosomatic symptoms in large-scale community studies [7].

Adolescents' smoking and binge drinking were assessed by questioning the participants "How often do you currently smoke cigarettes (or tobacco)?" and "How many times (if any) did you have five or more drinks in a row in the past 30 days?" Answers were rated on a 4 and 5 points Likert scale respectively and were finally grouped into two categories ("smoking/no smoking" and "binge drinking/no binge drinking"). Socio-economic status was measured by the Family Affluence Scale[FAS; 9], a child/ adolescent-specific, self-reported indicator of family wealth, that was categorized into three groups in the analysis [low level (0-3), intermediate (4-5) and high level (6-7)]. 


\section{Statistical Analysis}

The chi-square test was used to compare the two groups of psychosomatic symptom levels in terms of smoking, binge drinking, socioeconomic status, age and gender. In order to evaluate the multiple effects of independent variables on high levels of psychosomatic symptoms, a multiple logistic regression model was constructed using a stepwise procedure. Adjusted odds ratios with 95\% confidence intervals were computed. Model diagnostics were evaluated using the Hosmer and Lemeshow statistic. All tests were two-sided, at a significance level of $P<0.05$. Analyses were conducted using SPSS statistical software (version 19.0) (SPSS, Chicago, IL, USA).

Table 1. Psychosomatic symptoms, smoking, binge drinking and socio-demographic characteristics.

\begin{tabular}{|c|c|c|c|c|c|}
\hline & \multicolumn{4}{|c|}{ Psychosomatic symptoms } & \multirow{3}{*}{$P$ value } \\
\hline & \multicolumn{2}{|c|}{ Low levels } & \multicolumn{2}{|c|}{ High levels } & \\
\hline & $\mathrm{N}$ & $\%$ & $\mathrm{~N}$ & $\%$ & \\
\hline \multicolumn{6}{|l|}{ Gender } \\
\hline Girls & 337 & 48,1 & 361 & 51,9 & \multirow{2}{*}{$<0.001$} \\
\hline Boys & 295 & 63,4 & 170 & 36,6 & \\
\hline \multicolumn{6}{|c|}{ Age (years) } \\
\hline $12-15$ & 457 & 57,9 & 332 & 42,1 & \multirow{2}{*}{$<0.001$} \\
\hline $16-18$ & 172 & 46 & 202 & 54 & \\
\hline \multicolumn{6}{|c|}{ Socio-economic status } \\
\hline Low & 221 & 50,5 & 217 & 49,5 & \multirow{3}{*}{0.210} \\
\hline Medium & 292 & 56,2 & 228 & 43,8 & \\
\hline High & 107 & 52,2 & 98 & 47,8 & \\
\hline \multicolumn{6}{|l|}{ Smoking } \\
\hline No & 594 & 56,9 & 450 & 43,1 & \multirow{2}{*}{$<0.001$} \\
\hline Yes & 38 & 31,9 & 81 & 68,1 & \\
\hline \multicolumn{6}{|c|}{ Binge drinking } \\
\hline No & 465 & 57,8 & 341 & 42,2 & \multirow{2}{*}{$<0.001$} \\
\hline Yes & 163 & 45,7 & 194 & 54,3 & \\
\hline
\end{tabular}

\section{Results}

Table 1 illustrates the differences between the two groups of psychosomatic symptom levels in terms of smoking, binge drinking and their socio-demographic characteristics. The multiple logistic regression analysis (Table 2) showed that the odds ratio for high levels of psychosomatic symptoms was significantly higher for girls and adolescents who have reported practicing smoking or binge drinking in comparison to boys and peers who abstain from these health-risk behaviours. Adolescents' age and socio-economic status did not appear to be robust predictors of high levels of psychosomatic symptoms.

Table 2. Multiple logistic regression analysis with high levels of psychosomatic symptoms as the dependent variable.

\begin{tabular}{|c|c|c|}
\hline & OR $(95 \% \mathrm{Cl})$ & $P$ value \\
\hline \multicolumn{3}{|l|}{ Gender } \\
\hline Boys & $1.00 *$ & \multirow{2}{*}{$<0.001$} \\
\hline Girls & $1.93(1.49-2.5)$ & \\
\hline \multicolumn{3}{|c|}{ Age (years) } \\
\hline $12-15$ & $1.00 *$ & \multirow{2}{*}{0.087} \\
\hline $16-18$ & $1.27(0.97-1.68)$ & \\
\hline \multicolumn{3}{|c|}{ Socio-economic status } \\
\hline Low & $1.00 *$ & \\
\hline Medium & $0.89(0.68-1.17)$ & 0.414 \\
\hline High & $1.12(0.78-1.6)$ & 0.545 \\
\hline \multicolumn{3}{|l|}{ Smoking } \\
\hline No & $1.00 *$ & \multirow{2}{*}{$<0.001$} \\
\hline Yes & $2.31(1.45-3.68)$ & \\
\hline \multicolumn{3}{|c|}{ Binge drinking } \\
\hline No & $1.00 *$ & \multirow{2}{*}{0.005} \\
\hline \multirow[t]{2}{*}{ Yes } & $1.49(1.13-1.98)$ & \\
\hline & *indicates refe & ce categor \\
\hline
\end{tabular}




\section{Discussion}

Our study is one of the few to depict associations between psychosomatic symptoms, smoking and binge drinking practices in a nation-wide, large, random, school-based adolescent sample. Corroborating previous research $[2,3,8]$, our study showed that adolescents with smoking and binge drinking practices had an elevated risk for multiple, recurrent psychosomatic symptoms, revealing a significantly impaired psychosomatic functioning compared to abstainers. However, the cross-sectional design of our study could not determine any causal relationships. It may be suggested that adolescents' psychosomatic ailments, smoking and binge drinking practices could be indicative of an underlying psychosocial distress with adverse effects on individuals' health and achievements that may extend to adult years $[1,10]$. Future research should examine the role of adolescents' coping skills and affect regulation in the relationship between psychosomatic symptoms and health-risk behaviours.

\section{Conclusion}

Our findings could inform adolescent health practices, so as aspects of health-risk behaviours are explicitly assessed among adolescents with high levels of psychosomatic symptoms who attend school and community health services. Early identification of adolescents with co-occurring health-risk behaviours and noticeable psychosomatic disturbances could flag individuals with a severe burden of psychosocial distress and, therefore, facilitate appropriate referrals and tailored interventions.

\section{Acknowledgements}

The KIDSCREEN project was financed by a grant from the European Commission (QLG-CT-2000-00751) within the EC 5th Framework-Programme "Quality of Life and Management of Living Resources". It was coordinated by Prof. Ulrike Ravens-Sieberer, Head of Research - Professor for Child Public Health at the University Medical Center HamburgEppendorf. This research has been co-financed by the European Union (European Social Fund - ESF) and Greek national funds through the Operational Program "Education and Lifelong Learning" of the National Strategic Reference Framework (NSRF) Research Funding Program: Heracleitus II. Investing in knowledge society through the European Social Fund.

\section{References}

1. Laukkanen E, Shemeikka S, Notkola I-L, Koivumaa-Honkanen H, Nissinen A. Externalizing and internalizing problems at school as signs of health damaging behavior and incipient marginalization. Health Promot Int. 2002; 17(2): 139-46.

2. Ghandour RM, Overpeck MD, Huang ZJ, Kogan MD, Scheidt PC. Headache, Stomachache, Backache, and Morning Fatigue Among Adolescent Girls in the United States. Associations With Behavioral, Sociodemographic, and Environmental Factors. Arch Pediatr Adolesc Med. 2004; 158: 797-803.

3. Simpson K, Janssen I, Boyce W, Pickett W. Risk taking and recurrent health symptoms in Canadian adolescents. Prev Med. 2006; 43(1): 46-51.

4. Currie C, Zanotti C, Morgan A, et al., eds. Social determinants of health and well-being among young people. Health Behaviour in School-aged Children (HBSC) study: international report from the 2009/2010 survey. Copenhagen: WHO Regional Office for Europe; 2012.

5. Giannakopoulos G, Tzavara C, Dimitrakaki C, Kolaitis G, Rotsika $\checkmark$, Tountas Y. Emotional, behavioural problems and cigarette smoking in adolescence: findings of a Greek cross-sectional study. BMC Public Health. 2010; 10: 57.

6. Currie C, Samdal O, Boyce W, Smith R, editors. Health Behaviour in School-Aged Children: a WHO Cross-National Study (HBSC): Research Protocol for the 2001/2002 Survey. Edinburgh: Child and Adolescent Health Research Unit, University of Edinburgh; 2001. 
7. Ottova V, Erhart M, Vollebergh W, Kökönyei G, Morgan A, Gobina I, et al. The Role of Individual- and Macro-Level Social Determinants on Young Adolescents' Psychosomatic Complaints. J Early Adolesc. 2012; 32(1): 126-58.

8. Botello-Harbaum M, Haynie D, Murray K, Iannotti R. Cigarette smoking status and recurrent subjective health complaints among US school-aged adolescents. Child Care Health Dev. 2011; 37(4): 551-8.

9. Currie CE, Elton RA, Todd J, Platt S. Indicators of socioeconomic status for adolescents: the WHO health behaviour in schoolaged children survey. Health Educ Res 1997; 12(3): 385-97.

10. Poikolainen K, Aalto-Setala T, Marttunen M, Tuulio-Henriksson A, Lonnqvist J. Predictors of somatic symptoms: a five year follow up of adolescents. Arch Dis Child. 2000; 83: 388-92.

\section{Comment on this article:}

\section{(f) (B) in 8 \& $P$}

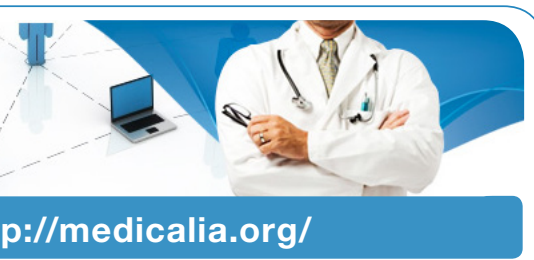

\section{http://medicalia.org/}

Where Doctors exchange clinical experiences, review their cases and share clinical knowledge. You can also access lots of medical publications for free. Join Now!

\section{Publish with iMedPub}

\section{http://www.imed.pub}

International Archives of Medicine is an open access journal publishing articles encompassing all aspects of medical science and clinical practice. IAM is considered a megajournal with independent sections on all areas of medicine. IAM is a really international journal with authors and board members from all around the world. The journal is widely indexed and classified Q1 in category Medicine. 